\title{
Laparoscopic Cholecystectomy in a Hostile Abdomen, is it Feasible
}

\author{
A.AYusuf, E.M.Oraby, A,K.Allam and M.G.Ebeed
}

General Surgery Dept., Faculty of Medicine, Benha Univ., Benha, Egypt

E-Mail:Mohamed@gmail.com

\begin{abstract}
The appearance of laparoscopic cholecystectomy has been a critical achievement in the treatment of gallstone infection, yet in the development of careful treatment toward the negligible access approach. The point of this examination was to survey the plausibility and security of laparoscopic cholecystectomy in patients with an antagonistic midsection for example patients with past upper stomach medical procedure. This examination was carried on 30 patients who had past significant upper stomach medical procedure, with indicative cholelithiasis, barring patients with intense cholecystitis, basic bile conduit infection, and current pancreatitis. The patients were 13 guys $(43.3 \%)$ and $17(56.7 \%)$ females. Their ages went between $29-75$ years with a mean of $43.33 \pm 11.41$. All patients had upper stomach medical procedures, 16 of them (53.3\%) had hernioplasty (13 of them were paraumbilical, one umbilical, one epigastric and one incisional). 9 cases (30\%) had investigation, 3 had splenectomy (10\%) and 2 had sleeve gastrectomy (6.7\%). Attachments were found at the employable field in 9 cases (30\%), at port locales in 5 cases (16.7\%), at both the usable field and port destinations at 13 cases $(43.3 \%)$ and away from the usable field for example not meddling with perception of the usable field in 3 cases $(10 \%)$. The usable time ran somewhere in the range of 45 and 75 minutes with a mean of $58.0 \pm 8.17$. Laparoscopic cholecystectomy can be performed securely in patients with an antagonistic mid-region, It is the helpful methodology of decision for gallstones illness in patients with a background marked by past upper stomach medical procedure.
\end{abstract}

Keywords: Laparoscopic, Cholecystectomy, Hostile Abdomen.

\section{Introduction}

The appearance of laparoscopic cholecystectomy has been a huge achievement in the treatment of gallstone sickness, yet in the advancement of careful therapy toward the insignificant access approach, the point of which is proposed to limit the injury of access without trading off the introduction of the careful field [1].

The first laparoscopic cholecystectomy performed on a human patient was done in 1987 by the French doctor Mouret. The fast acknowledgment of the strategy of laparoscopic medical procedure by everyone is unmatched in careful history. It has changed the field of general medical procedure more definitely and more quickly than some other careful achievement [2].

Laparoscopic cholecystectomy is currently viewed as the best quality level of treatment for indicative cholelithiasis and ongoing cholecystitis [3].

Laparoscopic cholecystectomy has certain points of interest over the open cholecystectomy methodology, for example, a more limited medical clinic remain, quicker re-visitation of every day exercises, and lower horribleness and death rates, both in suggestive cholelithiasis and intense cholecystitis. Because of new advancements in laparoscopic instrument innovation and improved laparoscopic experience of the specialists, laparoscopy is proceeding to develop. At first, pregnancy, corpulence, cirrhosis, and past stomach medical procedure were acknowledged as relative contraindications for laparoscopic cholecystectomy. Moreover, past stomach medical procedure is accounted for to build the confusion rate and delay emergency clinic remain after laparoscopic cholecystectomy [4].

The objective of each activity is to distinguish the important pathologic condition, evade injury to the intrastomach substance, and satisfactorily close the stomach divider. These destinations can be especially testing to accomplish in patients with a "threatening" mid-region.
An antagonistic midsection is commonly characterized as one in which admittance to the pathologic state of interest is obstructed or troublesome due to trouble crossing the stomach divider or monstrous intra-stomach bonds [5].

Initially, patients with past stomach medical procedure were prescribed not to go through laparoscopy on account of the expanded danger of entering entrail injury brought about by Veress needle or first trocar inclusion through inside disciple to the stomach divider. Additionally, laparoscopic adhesiolysis performed to increase satisfactory introduction to the usable field was dreaded to be additional tedious and possibly more deceptive than open adhesiolysis [6].

Thirty to half of patients introducing for laparoscopic cholecystectomy have history of past stomach medical procedure and $60 \%$ of these require adhesiolysis. Patients with past stomach medical procedure present two explicit issues: Obtaining safe admittance to the stomach depression to accomplish the pneumoperitoneum and playing out a safe adhesiolysis to increase satisfactory presentation to the usable field. Obstruction with access relies unequivocally upon the area of the past medical procedure [7].

Most vascular wounds are related with a visually impaired inclusion procedure of the primary port, while the greater part of all gut wounds are related with this strategy. The dangers for inside injury or vascular injury are considerably higher if the needle is aimlessly positioned through a past entry point. The Veress needle created by Veress in 1938 for formation of pneumothorax for the treatment of pneumonic tuberculosis is utilized to make the pneumoperitoneum. Frequency of umbilical grips might be as high as $68 \%$ in patients with past stomach medical procedure particularly in those where a midline scar reaches out to the umbilical district. More secure options incorporate 
arrangement of the needle and first trocar at a site a long way from the past scar, this permits improved capacity to see the stomach depression since vision isn't clouded by bonds and better evaluation of area of outstanding ports should be possible and specialists have suitable working separation important to control instruments. Palmer's point, found $3 \mathrm{~cm}$ sub-par compared to the subcostal curve in the left mid clavicular line is a mainstream safe other option. Past stomach medical procedure isn't a contraindication to endeavoring a system laparoscopically. Patients ought to be cautioned of the expanded danger for entrail injury, the conceivable requirement for extra trocars and expanded danger for transformation [8].

The point of this examination is to evaluate the practicality of laparoscopic cholecystectomy in patients with an antagonistic midsection as in past stomach medical procedure, taking a gander at the dismalness of the technique, change rate and other perioperative complexities.

\section{Patients and methods}

Patients were recruited from the outpatient clinic and emergency department at Benha University Hospital and Benha Insurance Hospital. This prospective study includes 30 patients with gall stones, documented by ultrasonography, undergoing laparoscopic cholecystectomy, in the period between June 2019 and April 2020. All patients have history of previous abdominal surgery.

\subsection{Inclusion criteria}

- Both genders are included.

- Age group: Any age.

- Patients proved to have gall bladder stones as the primary cause of their complaint.

- Patients with history of a cause of a hostile abdomen, i.e. previous abdominal surgery.

\subsection{Exclusion criteria}

- Patients unfit for general anesthesia.

- Patients unfit for pneumoperitoneum (e.g. increased intracranial pressure, hemodynamic instability, severely cardiopulmonary-compromised patients, etc).

- Patients with associated common bile duct disease.

- Patients with portal hypertension.

- Patients with severe coagulopathy.

- Patients with generalized peritonitis.

- Patients with acute pancreatitis.

- Patients with acute cholecystitis.$$
\bullet
$$

\subsubsection{Pre-operative assessment}

All patients were worked up thoroughly and were subjected to detailed history including presenting symptoms; duration of symptoms, previous surgery, and chronic illness. All patients had general physical examination, abdominal examination and systemic examination. And investigations as:

- Complete blood count.

- Liver function tests.

- Kidney function tests.

- Coagulation profile.

- Viral markers (Hepatitis B and C).

- ECG if needed.

- Abdominal ultrasonography.

\subsubsection{Operative data}

Trocar situation was arranged by the presence of scars, particularly the first trocar, on the grounds that increasing a sheltered section into the peritoneal hole is of most extreme significance. In patients with past umbilical or paraumbilical hernia fix (14 patients), the first trocar was embedded in the subxiphoid district. In patients with scars in the left upper stomach quadrant (for example past splenectomy, 3 patients), the first trocar was embedded periumbilical. In patients with a midline cut (10 patients), the first trocar was embedded either periumbilical (a few centimeters substandard or horizontal to the umbilicus) or at Palmer's point, as per the degree of the scar and the normal destinations of bonds. Subsequent to increasing a sheltered admittance, different ports were presented under direct vision.

We utilized the ordinary four port strategy, utilizing the North American plan, with the working specialist remaining on the patient's left, careful collaborator on the patient's privilege and clean tech/nurture on the patient's left, and the patient lying recumbent. The video screen was set on the patient's correct. The hardware utilized incorporated a video screen, one laparoscope $(5 / 10 \mathrm{~mm}$, $0 / 30$ degrees) including the camera line and a light source, carbon dioxide source and tubing for insufflation, $5 \mathrm{~mm}$ to $10 \mathrm{~mm}$ trocars, laparoscopic instruments, for example, atraumatic graspers, Maryland grasper, cut applier and electrocautery (e.g., snare, spatula). We additionally utilized a surgical tool (11/15 sharp edge), forceps, needle driver, and absorbable stitches. A significant open plate was readied, for conceivable change.

Post-usable note was made for the necessity of channel addition or not, span of the channel, term of clinic remain, season of oral admission and time to revisitation of full action. Post-employable absense of pain was finished with nonsteroidal mitigating drug (Diclofenac) in the recuperation room and any extra portion if essential.

\section{Results}

Data was collected and analyzed as follows: Table (1) shows the gender and age distribution among the studied patients. 13 patients $(43.3 \%)$ were males, while 17 (56.7 $\%)$ were females. Their ages ranged between $29-75$ years with Mean \pm SD $(43.33 \pm 11.41)$ as shown in Table (1) 
Table (1) Distribution of the studied cases according to demographic data $(n=30)$.

\begin{tabular}{lll}
\hline & No. & \% \\
\hline Sex & & \\
Male & 13 & 43.3 \\
Female & 17 & 56.7 \\
Age (years) & & \\
$\mathbf{4 0}$ & 16 & 53.3 \\
$\mathbf{7 0}$ & 14 & 46.7 \\
Min. - Max. & $29.0-75.0$ & \\
Mean $\mathbf{\text { SD. }}$ & $43.33 \pm 11.41$ & \\
Median (IQR) & $40.0(35.0-48.0)$ & \\
\hline
\end{tabular}

Table (2) also shows the distribution of the patients according to history of comorbidities where 12 patients (40\%) have history of chronic diseases distributed as follows: 9 patients with DM (30\%), 7 with HTN (23.3\%) and 2 with $\mathrm{HCV}(6.7 \%)$. Table (2) also shows the distribution of patients according to previous ERCP. The vast majority (90\%) have no history of previous ERCP, while only $10 \%$ had ERCP.

Table (2) Distribution of the studied cases according to different parameters $(n=30)$.

\begin{tabular}{lll}
\hline & No. & $\%$ \\
\hline Previous abdominal surgery & & \\
Hernioplasty & 16 & 53.3 \\
Exploration & 9 & 30.0 \\
Sleeve gastrectomy & 2 & 6.7 \\
Splenectomy & 3 & 10.0 \\
Chronic diseases & & \\
No & 18 & 60.0 \\
Yes & 12 & 40.0 \\
DM & 9 & 30.0 \\
HTN & 7 & 23.3 \\
HCV & 2 & 6.7 \\
Previous ERCP & & \\
No & 27 & 90.0 \\
Yes & 3 & 10.0 \\
\hline
\end{tabular}

Sites of adhesions: Adhesions were present at the operative field in 9 cases $(30 \%)$, at port sites in 5 cases (16.7\%), at both the operative field and port sites at 13 cases $(43.3 \%)$ and away from the operative field i.e. not interfering with visualization of the operative field in 3 cases (10\%) Fig (1).

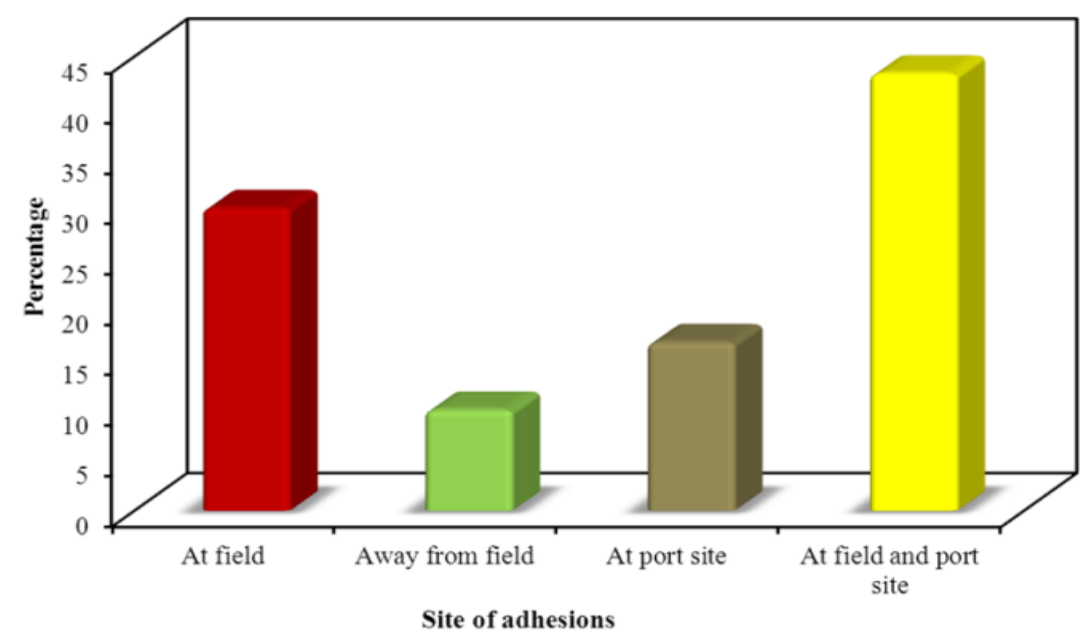

Fig (1) Distribution of the studied cases according to Site of adhesions ( $n=30)$. 
Need for adhesiolysis: Most of the studied cases (23 cases, $76.7 \%$ ) required adhesiolysis, while in 7 of them
(23.3\%) adhesiolysis was not needed Fig (2).

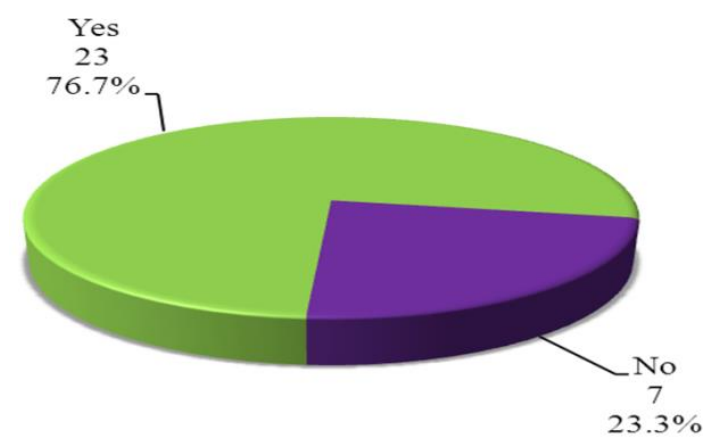

Fig (2) Distribution of the studied cases according to Adhesiolysis needed $(n=30)$.

Table (3) shows the time needed for return to full activity, which ranged between 3-7 days, with Mean \pm SD (4.77 \pm $1.33)$.

Table (3) Distribution of the studied cases according to hospital stay and time of return to full activity $(n=30)$.

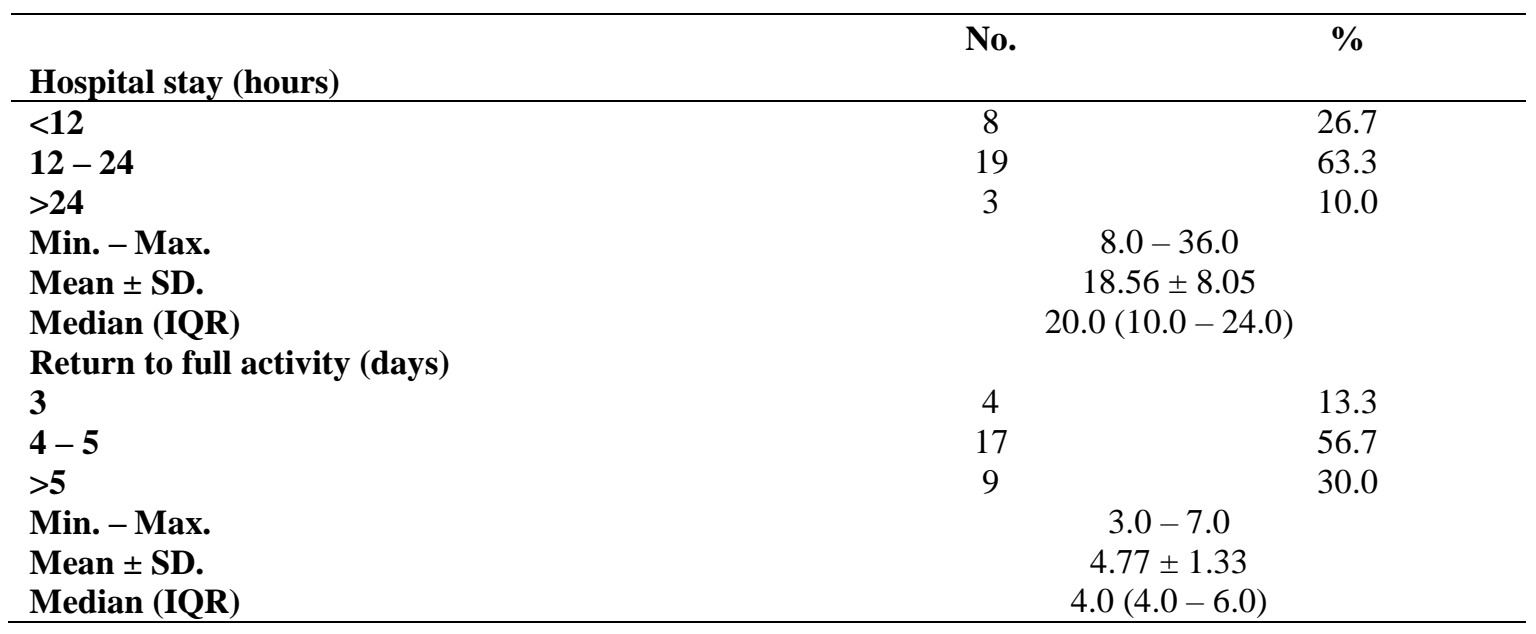

\section{Discussion}

During medical procedure, the specialist must fight the temptation to unnecessarily lyse bonds. Just those attachments that genuinely meddle with representation of the employable field or that would forestall the position of ensuing trocars under vision ought to be lysed. In our investigation, attachments were found in all patients. Notwithstanding, adhesiolysis was needed in 23 patients (76.7\%). Minor draining was straightforwardly owing to adhesiolysis. As we would like to think, most of bonds from earlier stomach medical procedure don't modify the life systems of the correct upper stomach quadrant and don't contrarily affect the exhibition of an effective laparoscopic cholecystectomy. Our general laparoscopic achievement rate in patients with past stomach medical procedure was $100.0 \%$, transformation rate was $0 \%$. In any case, patients who had gone through stomach medical procedure had expanded trouble during laparoscopic cholecystectomy because of essence of bonds in the upper midsection, which thusly prompted longer employable occasions as a rule, likely because of the requirement for adhesiolysis.
No compelling reason to make reference to that there is not a viable replacement for fastidious dismemberment, recognizable proof and show of different crucial structures close to the basic bile pipe. Considering this idea, the specialist must be careful with a couple of anatomical tourist spots for safe analyzation so as to evade bile pipe injury. For instance, he should remain nearby to the liver edge, either medially or along the side, to search for and recognize the gallbladder under the grips. He ought to recognize the gallbladder neck. This permits perception and appropriate presentation of the cystic supply route at the degree of the gallbladder neck. He should then distinguish the intersection between the gallbladder and the cystic conduit. Dismemberment of the cystic pipe and cystic corridor starts contiguous or close to the point of beginning of cystic conduit or close to the point of the vessel passage. The lymph hub of Lund is a significant milestone for characterizing the cystic conduit and course. Dismemberment of Calot's triangle should begin simply after the intersection between the gallbladder and cystic conduit has been recognized. Rouviere's sulcus is 
another significant milestone. This 2 to $5 \mathrm{~cm}$ long sulcus hurries to one side of the liver hilum front to the caudate cycle and normally contains the correct entrance group of three or its branches. The sulcus was found to precisely show the plane of basic bile pipe. Legitimate limitation of the normal bile channel every now and then is significant during medical procedure. This is cultivated by withdrawing the duodenum descending, remembering the plane of Rouviere's sulcus and withdrawing the correct flap of liver with appropriate foothold on Hartmann's pocket. At the point when it is hard to imagine the gallbladder in thick fibrotic attachments to the liver, as in instances of patients with past upper stomach medical procedure, the best methodology is to remain nearby to the liver edge and calmly go on with adhesiolysis, holding the bonds near the liver edge and taking consideration not to pull them from the liver case. [9].

It is significant for the specialist to comprehend that transformation to an open methodology is neither a disappointment nor an inconvenience, however is an endeavor to guarantee patient's wellbeing, and the equivalent ought to be disclosed to the patient before medical procedure.

In our examination, difficulties happened uniquely in 3 patients (10\%) as minor seeping from adhesiolysis.

Ongoing investigations uncover that intense cholecystitis, pancreatitis, and basic bile pipe stones are factors that may prompt transformation to an open methodology and contrarily influence medical clinic remain, usable time, and perioperative entanglement rates. We rejected such cases so as to decide the right and target likelihood of transformation to an open system in patients with past stomach medical procedure. The primary motivation behind this choice was to have a gathering of patients with homogenous conditions or factors. On the off chance that we had incorporated these patients, conditions like pancreatitis, intense cholecystitis, and basic bile channel stones would have influenced the transformation rate, usable time, change rate, and perioperative difficulty rate. Clinic remain of these barred patients would likewise have been longer. When prohibited patients were assessed, adding past upper stomach medical procedure to any of these avoided factors demonstrated an expansion in the perioperative complexity rate, the mean employable time, and the mean postoperative clinic remain. Consequently, these variables were avoided, else it would have indicated factually huge contrast. [10].

A few investigations propose that in men with indicative gallbladder stones, irritation and fibrosis happen more frequently than they do in ladies with a similar illness. This converts into troublesome dismemberments during laparoscopic cholecystectomy and a higher pace of change to open medical procedure in male patients. Nonetheless, we didn't see any critical distinction among people in our investigation. [11].

Age didn't affect the consequences of our examination. We found no distinction among youthful and old patients in our investigation in regards to any of the exploration standards, which is predictable with different examinations. For instance, Lill; et al. expressed in their investigation that there were no measurably critical contrasts in mean usable time or transformations to open medical procedure between the age gatherings. The mean hospitalization time and intricacies expanded with the age of the patients more than 75 years. Neither extreme inconveniences nor mortality were experienced. The most seasoned patient in our investigation was 75 years of age. [12].

In our investigation, no wounds happened during port arrangement, we had no trouble in formation of pneumoperitoneum. No iatrogenic wounds to inside circles, significant veins or different structures happened. There were no nerve bladder hole, normal bile pipe injury, or warm injury. There was no uncontrolled dying, just minor seeping from adhesiolysis in 3 patients (10\%) which was controlled with cloth, pressure and diathermy without need to change over to an open strategy. No affected stones in the bile conduits were found. Circumstances where trouble in gallbladder extraction or instrument disappointment requiring change didn't emerge. There were no passings revealed.

\section{Conclusion}

Laparoscopic cholecystectomy can be performed securely in patients with an antagonistic midsection, for example past upper stomach medical procedure, however it is related with an expanded requirement for adhesiolysis and, here and there, a more extended usable time. It is the helpful technique of decision for gallstones illness in patients with a background marked by past upper stomach medical procedure.

\section{References}

[1] A.Cuschieri . The european experience with laparoscopic cholecystectomy," Am. J. Surg., Vol. 161, no. 3, PP.385-387, doi: 10.1016/00029610(91)90603-B,1991.

[2] S.J.Spaner , G.L.Warnock. A Brief History of Endoscopy, Laparoscopy, and Laparoscopic Surgery," J. Laparoendosc. Adv. Surg. Tech, Vol.7(6), PP.369-373, 1997.

[3] F.Coccolini. Open versus laparoscopic cholecystectomy in acute cholecystitis. Systematic review and meta-analysis," Int. J. Surg., Vol. 18, PP.196-204, doi: 10.1016/j.ijsu.2015.04.083,2015.

[4] D. Atasoy, A.Aghayeva, I.Sapc. Effects of prior abdominal surgery on laparoscopic cholecystectomy," Turkish J. Surg, Vol.34(3), PP.217-220, doi: 10.5152/turkjsurg. 3930,2017.

[5] S.R.Steele. Complications, Considerations and Consequences of Colorectal Surgery, An Issue of Surgical Clinics, 1st ed. Elsevier Health Sciences, Vol.4(1), PP.40-73, 2013.

[6] D J.Bowrey. SPECIAL PROBLEMS IN Previous Abdominal Surgery ," Surg. Endosc., Vol. 80(4), p. 52242, 2000. 
[7] A.Assalia, M.Gagner, M.Schein. Controversies in Laparoscopic Surgery. Berlin, Heidelberg: Springer, Vol.2(3), PP.54.87, 2006.

[8] E.Ritesh Kohli, A.K.Bansal, P.S.Gupta. A study of laparoscopic cholecystectomy in patients with previous abdominal surgery," Med. Res. Chronicles. Vol. 1(2), PP.130-140, 2014.

[9] K.Singh, A.Ohr. Anatomic landmarks: their usefulness in safe laparoscopic cholecystectomy," Surg. Endosc. Other Interv. Tech. Vol. 20(11), PP.1754-1758, 2006.
[10] A.Alponat, C.K.Kum, B.C.Koh. Predictive factors for conversion of laparoscopic cholecystectomy," World J. Surg. Vol. 21(6), PP.629-633, 1997.

[11] S.Yol, A.Kartal, C.Vatansev. Sex as a factor in conversion from laparoscopic cholecystectomy to open surgery," JSLS J. Soc. Laparoendosc. Surg. Vol. 10 (3), p. 359, 2006.

[12] S.Lill, A.Rantala, T. Vahlberg. Elective laparoscopic cholecystectomy: the effect of age on conversions, complications and long-term results," Dig. Surg., Vol. 28(3), PP.205-209, 2011. 